\title{
0923 ENHANCING SURVEILLANCE STRATEGIES FOR CHILDHOOD SELF-REPORTING DATA
}

S D Jepsen*, J R Wilkins, J Mac Crawford, K M Koechlin, T L Bean Correspondence: Department of Preventative Medicine, The Ohio State University, 590 Woody Hayes Drive Columbus, $\mathrm{OH} 43210,43210$, USA

\subsection{6/ip.2010.029215.923}

Problem Investigators routinely incorporate youth self-report data into cohort studies with variable results. Our study successfully employed strategies to ensure data quality and reduce participant fatigue.

Methods A longitudinal study of rural youth aged 9-18 years was conducted to develop multivariable risk prediction models 


\section{IP Safety 2010 abstracts}

of agricultural related injuries at the same time collecting unintentional injury data for children exposed to agricultural hazards. Over 400 youth were involved in a 13-week cohort study. During this time period, each person interacted with researchers in a multitude of ways to remain engaged in the study, avoid reporting fatigue, and deliver quality self-reported data.

Results To obtain study results, youth (and their consenting parents) received letters of invitation. Once enrolled, the study team met face-to-face with each subject to describe the reporting process. Complimentary to completing a daily record book, youth had weekly interaction with an electronic toll free phone system. If the youth did not phone in, the system called them until contact was made. Additional incentives included a personal wristwatch, monetary incentives for each week data was submitted, and lottery opportunity to win sport tickets.

Conclusion Semilogarithmic plots of rates of all unintentional injuries (US data from 2000) as well as agriculture-related injuries (US and Canadian data from 19 previous studies) graphed as a function of injury severity exhibited linearity. Our cohort reported injury rates 1.4 to 4.3 times higher than national rates, suggesting that our methodology can significantly reduce injury under-reporting. 